\title{
Clinical aspects of ' $Q$ ' fever
}

\author{
G. LAING BROWN \\ M.D., D.P.H. \\ Bucknall Hospital, Stoke-on-Trent, Staffordshire
}

\begin{abstract}
Summary
' $Q$ ' fever is an acute febrile illness seen mainly among members of the farming community. The infection is contracted from sheep and cows mainly by inhalation of infected dust, but can be spread by raw milk.

The symptoms bear a marked resemblance to influenza and in the majority of cases the illness clears up spontaneously. A firm diagnosis can best be made by demonstrating a rise in complement fixing antibodies. Patchy consolidation of the lungs is seen on $X$-ray in about one-third of the cases and occasionally the infection becomes implanted on previously damaged heart valves and gives rise to subacute bacterial endocarditis. Treatment with tetracycline or trimethoprim sulphamethoxazole is indicated.
\end{abstract}

IN ' $Q$ ' fever man's reaction to infection is fundamentally different from that of the animals from which he acquires his infection.

Sheep and cows show no apparent clinical symptoms and the organism, though present in high concentration in the udder, does not interfere with milk production. Man, on the other hand, reacts to infection by developing an acute febrile illness.

Sporadic cases occur in North Staffordshire where there are a large number of hill farms whose farmers live on the verge of poverty-they have a few hens, two or three cows, and some sheep on the hillside. The milk is not pasteurized because there is enough only to serve the needs of the farmer and his family and a few of his neighbours - the opportunity, therefore, of milk spread of infection is ever present.

Interest in the subject was stimulated in 1967 by a fortuitous outbreak of twenty-nine cases in a detention centre which was investigated in some detail.

The majority of cases of ' $Q$ ' fever are never diagnosed as such. Many people with antibodies in the blood can recall a febrile illness which was diagnosed as 'flu' and from which they recovered in a few daysproof is lacking but for the associated history which led to investigation for rising antibodies. The patient's occupation has some bearing on how thorough is the investigation-veterinarians, farmers and abattoir workers, for example, usually get investigated in depth.
The average case is admitted as a 'flu-like illness' and a firm diagnosis is only made in retrospect after a second blood sample taken after 14 days shows a rising level of antibodies by which time the patient has recovered and is back at work.

The farmer of today and his country G.P. are very conscious of the possibility of brucellosis and many such cases are referred for diagnosis and treatment. It is among this group that the occasional case of ' $Q$ ' fever sometimes turns up.

In a typical case the onset is sudden with a temperature ranging from 38 to $40.5^{\circ} \mathrm{C}$ and accompanied by severe sweating, shivering and backache. In addition most patients complain of muscular pains in the limbs and headache. The throat is often red and inflamed, but exudate on the tonsils is not a feature. Photophobia and suffusion of the conjunctiva are common. At least half the patients develop a non-productive but troublesome cough. In a minority sputum may develop later and this is very occasionally blood stained.

Clinical examination of the chest in the early stages is usually negative, but minimal signs of localized consolidation may be detected later. Routine chest X-rays are indicated in all patients with a cough because frequently single or multiple soft shadows are seen, especially in the lower lobes, despite the absence of physical signs.

It is stated that a Coxiella infection differs from a Rickettsia infection in that (a) it does not produce a Weil Felix reaction and (b) there is no rash. A mild generalized erythematous type of rash can occur, but a rash is certainly not a common feature of this disease.

The signs and symptoms and overall picture could well be the picture of a typical influenza or an attack of brucellosis. Only the laboratory can decide. Any laboratory asked to test for antibodies to brucellosis should automatically check for ' $Q$ ' fever antibodies. The two diseases are both prevalent in the same type of community.

Severe manifestations and complications of this disease

When patchy consolidation is present in the lungs it usually takes about 3 weeks to clear- 
sometimes it is more prolonged and pleural effusions sometimes develop.

If liver function tests are carried out minimal abnormalities are frequently detected, in particular a raised SGPT, but only very rarely is there a raised serum bilirubin or frank evidence of jaundice. One American study (Editorial, 1971) showed that if liver biopsies were carried out on all cases these showed extensive liver involvement despite only minimal abnormal findings in the liver function tests.

Headache and neck stiffness can be so severe that meningitis is suspected, but the CSF is invariably found to be quite normal (Report of the Public Health Laboratory Service, 1969). Lymphadenopathy is occasionally present and as sore throat is also a common symptom glandular fever has sometimes to be considered in the differential diagnosis. Pericarditis has been recorded where the patient has continued to run a lower grade fever over a period of several weeks.

In the acute stage a precise clinical diagnosis is difficult and the main conditions to be considered in the differential diagnosis are:

(1) Influenza. Especially in winter, when influenza is prevalent, an occasional case of ' $Q$ ' fever would be easily overlooked.

(2) Brucellosis. The sweating, the shivering, the aches and pains are common to both diseases and both conditions are most liable to occur in farming communities.

(3) Glandular fever. The sore throat and lymphadenopathy of ' $Q$ ' fever could well be confused with the early stages of glandular fever, but a blood picture and Paul Bunnell test will settle the question very quickly.

(4) Mycoplasma pneumoniae and other respiratory virus infections may have to be considered in cases where the respiratory symptoms and $\mathrm{X}$-ray evidence of patchy consolidation are the outstanding features.

(5) Psittacosis in the early stages also resembles ' $Q$ ' fever in that it often presents with a high temperature, shivering and sweating and a troublesome non-productive cough.

\section{Diagnosis}

A firm diagnosis can only be made with the assistance of the laboratory. Attempts at culture of the organism on the yolk sac of developing eggs or by guinea-pig innoculation present hazards of aerosol infection for the technicians. Many such cases of infection have been recorded and, therefore, such procedures are not justified for routine diagnosis. A four-fold or greater rise in titre of complement fixing antibodies is convincing evidence of recent infection.
Chronic Coxiella infection

Months or years after the acute attack a very small number of patients develop signs and symp- $\frac{a}{c}$ toms suggestive of sub-acute bacterial endocarditis. Blood cultures remain persistently negative, but if complement fixation tests are carried out usingo Phase 1 organisms the cause of the endocarditis is 음 quickly established. The infection is found on heart $\frac{\bar{m}}{\bar{b}}$ valves known to be previously damaged. In the $\overparen{\Phi}$ majority of cases it is the aortic valve which is involved, and for this reason the patient may sometimes present with a cerebral embolus.

\section{Treatment}

Tetracycline has been regarded for some years as the drug of choice. In the average acute case whether 3 . antibiotic is given or withheld appears to make little difference to the duration of pyrexia of the dis-ic appearance of symptoms. Analgesics and anti- $\rightarrow$ pyretics do much for the patient's comfort, and goodic nursing care with tepid sponging to reduce the pyrexia and changing of pyjamas when saturated음 with sweat are important measures in the acutestage.

Antibiotic should be administered to all suspectede

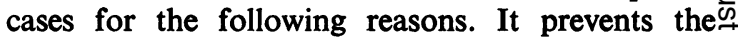
occasional case which goes on to develop a prolongedd $\vec{\theta}$ low grade pyrexia associated with pleurisy and pericarditis. It may likewise reduce the long tex risk of Coxiella endocarditis in those patients who already have damaged or congenitally deformed heart valves. It should reduce the risk of droplet spay spread from the patient to his nursing atten-市 dants.

Once endocarditis develops treatment, even withö $\overrightarrow{\vec{B}}$ prolonged courses of tetracycline, has produced 3 very disappointing results and in the majority of cases deterioration is progressive. Surgical intervention and replacement with an artificial valve gives윽 the best prospects for recovery. Antibiotic should:be given in the pre- and post-operative period.

Recent evidence suggests that trimethoprim sulphamethoxazole (Septrin) (Freeman \& Hodson, 1972) on its own or in combination with tetracycline may give better results, and this line of therapy? deserves more detailed investigation by workers in the field.

\section{Prophylaxis}

Although vaccines are available for cattle I sees little prospect for their success in the field of pre $\omega$ vention. The infection causes little upset, if any, in animals, it does not affect the milk yield of the cow, and has therefore no impact on the economics of 6 farming; the average farmer would have no interest in achieving a Coxiella-free herd because it does not affect his pocket. Brucellosis, which does affect the 
economics of dairy farming, is an example of how difficult it can be to persuade and cajole the farming industry to co-operate in the prevention of infections spreading from animals to man.

Heat treatment of all milk supplies seems to be the only answer as far as milk spread is concerned.

\section{References}

A Report from Five Laboratories of the Public Health Laboratory Service. (1969) The occurrence of Coxiella burneti in North West England and North Wales. Journal of Hygiene, 67, 125.

Editorial (1971) $Q$ fever and hepatitis. Journal of the American Medical Association, 216, 510.

Freeman, R. \& Hodson, M.E. (1972) Q fever endocarditis treated with trimethoprim and sulphamethoxazole. British Medical Journal, 1, 419. 